\title{
The Impact of Smart City Construction on the Quality of Foreign Direct Investment in China
}

\author{
Chong Ye, Zhaoyang Zhao $\mathbb{D}$, and Jinlong Cai $\mathbb{D}^{\circ}$ \\ School of Economics and Management, Fuzhou University, Fuzhou 350108, China \\ Correspondence should be addressed to Jinlong Cai; n190727092@fzu.edu.cn
}

Received 1 June 2021; Accepted 16 August 2021; Published 23 August 2021

Academic Editor: Miguel Fuentes

Copyright (C) 2021 Chong Ye et al. This is an open access article distributed under the Creative Commons Attribution License, which permits unrestricted use, distribution, and reproduction in any medium, provided the original work is properly cited.

\begin{abstract}
As a new model of urban development, it is important to scientifically analyze the impact of smart cities on the quality of foreign direct investment (FDI). This article constructs a difference in differences' model to test the impact of foreign direct investment quality in smart city construction based on panel data of 226 prefecture-level cities from 2006-2017. Study shows the following. (1) The construction of smart cities has significantly improved the quality of FDI utilized in pilot cities. (2) Heterogeneity analysis shows that smart city construction significantly contributes to the quality of FDI in the western city subsample and a subsample of cities with low natural resource dependence. After a series of robustness tests and placebo tests, the above conclusions still hold. The results of the study suggest that China should further promote the construction of smart cities to optimize the quality of foreign direct investment and promote high-quality economic growth. The findings of the study also provide theoretical and practical references for the construction of smart cities in China and other countries around the world.
\end{abstract}

\section{Introduction}

In 2008, the International Business Machines Corporation (IBM) puts forward the concept of smart city, China's smart city development in the concept of the introduction period; at this time, local enterprises are in the fumbling stage. The market is also dominated by international software system integrator. In 2012, with the increasing level of urbanization in China, the Ministry of Housing and Construction issued the "Interim Measures on $\mathrm{Na}$ tional Smart City Pilot," and the construction of smart cities in China started to get on track driven by the development of information technology. In 2016, as China's $5 \mathrm{G}$, big data, artificial intelligence (AI), and other technology levels become increasingly mature, China's smart city construction towards digital and intelligent development enters a new stage of development. As of early April 2020, the number of three batches of smart city pilot lists announced by the Ministry of Housing and Construction in China has reached 290. China's smart city construction has shown the trend of provincial-level cities leading, prefecture-level cities following, and county-level cities and city clusters starting, with emerging applications and models emerging in the development process.

Since China's accession to the World Trade Organization in 2001, the amount of foreign investment in China has shown an overall growth trend, with the actual amount of foreign investment utilized in China increasing from US\$ 53.505 billion in 2003 to US $\$ 163$ billion in 2020 (see Figure 1).As a result of the new crown epidemic, global FDI will total about $\$ 859$ billion in 2020, a significant contraction of $42 \%$ compared to 2019 , but China will absorb foreign investment against the trend of $4 \%$ growth, surpassing the United States to become the world's largest foreign investment inflow country. In the difficult time of global foreign investment flow, China achieves the "three improvements" in the total amount of attracted capital, growth rate, and global share. Global R\&D centers, headquarters projects, and supply chain operation centers have become hotspots for foreign investment in China. As a smart city in the first echelon of Shanghai, in January 15, 2021, 62 foreign investment projects concentrated in Shanghai, with a total investment of $\$ 11.85$ billion. And, the signed projects cover a number of fields such as artificial intelligence, integrated 


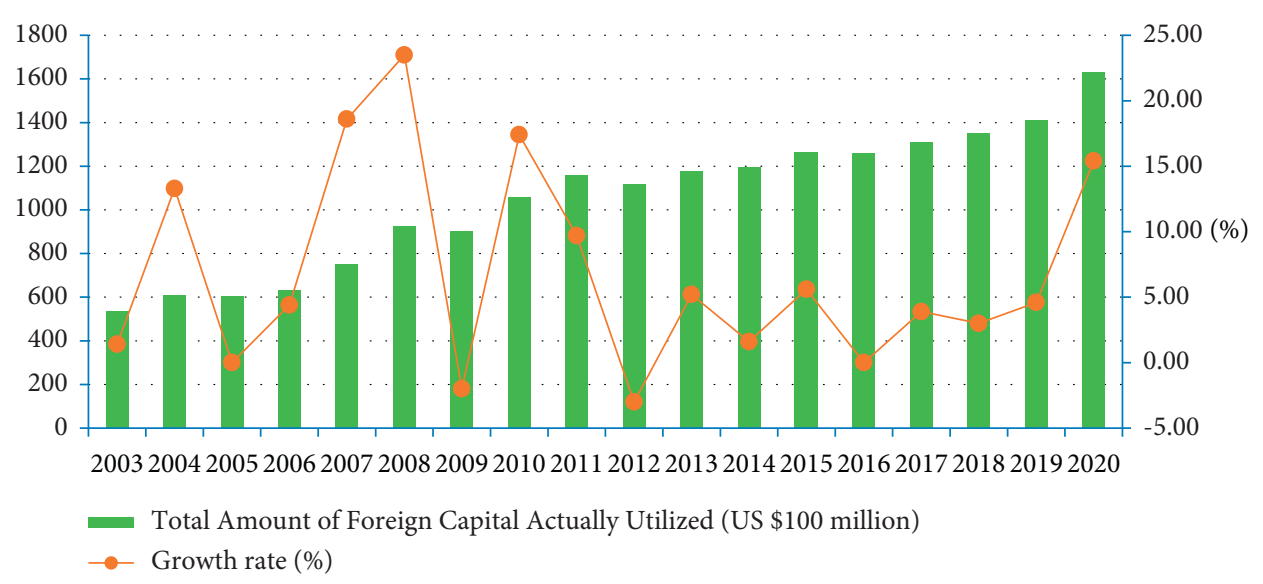

FIgURE 1: Total amount of foreign capital actually utilized in China from 2003 to 2020.

circuits, and digital economy, with the characteristics of high capacity of the investment body, key functional area projects, leading industry technology tip, and wide distribution of key industries. The comprehensive promotion of $s$ related to smart city innovation and reform trials will help promote the digital and intelligent transformation of industries in pilot cities and maintain the rapid development momentum of strategic emerging industries.

Although smart city construction has moved into the 2.0 era of data interconnection, there is little literature on the study of smart city pilots on the quality of foreign investment. In the context of the innovation-driven development strategy, the utility of specific applications of smart city construction is extensive and far reaching, especially in terms of transforming the city's economic development and thus improving the performance of attracting foreign investment. Only an in-depth investigation into the impact of China's smart city pilot construction on the quality of attracting foreign investment can provide targeted guidance for China's smart city construction practice on the use of foreign investment in cities under economic opening.

\section{Literature Review}

The research in this article is mainly related to the literature on three aspects: the significance of smart city construction, the mechanism of the role of smart city construction on China's high-quality development, and the study of factors influencing the quality of foreign investment.

2.1. The Significance of Smart City Construction. At present, scholars are mostly positive about the construction of smart cities. Alcaide Muñoz and Rodríguez Bolívar argue that smart city building can empower people in public decisionmaking and facilitate access to knowledge and innovationbased solutions for sustainable development [1]. Algorithms based on deep learning and clustering techniques, such as those of Parlina et al., argue that smart cities can be built in six dimensions of smart sustainability: technology, energy, environment, transportation, e-governance, human capital, and welfare [2]. Linde et al. argue that, in the smart city building environment, the requirements of technological development, digitization, and sustainability have led to an increasing dynamism of businesses and created new opportunities for innovation in urban systems [3]. Shi et al., using China as a case study, argue that the potential mechanism of smart city development inhibited the New Crown Pneumonia epidemic and that the epidemic has, to some extent, instead accelerate the construction of smart cities in China [4]. Interventionary studies involving animals or humans and other studies that require ethical approval must list the authority that provides approval and the corresponding ethical approval code.

\subsection{Mechanism of the Role of Smart City Construction on} China's High-Quality Development. The essence of a smart city is to use the Internet, big data, and other technical means to promote the organic coordination of urban service elements and residents' needs and to improve people's quality of life. At present, numerous scholars have focused on the research aspect of the effects of smart city construction. According to Zhou and Li, smart cities have a significant boosting effect on economic growth that increases with the level of urban human capital, higher levels of financial development, and lower levels of government intervention [5]. Tang empirically analyzed smart city construction as a proxy variable for the digital economy and concluded that it can promote high-quality urban development [6]. Shi and Zhang construct a PSM-DID model to empirically analyze a sample of 224 cities in China and find that smart city construction can significantly promote the development of the financial industry [7]. Nie reveals the logic of the "binary margin" expansion of smart city construction and foreign investment through empirical evidence from a panel of 186 prefecturelevel cities and argues that the expansion effect of foreign investment in smart city pilots is achieved mainly by reducing inputs and enhancing infrastructure construction [8].

2.3. Study on the Influencing Factors of Foreign Investment Quality. The earliest scholar to focus on the quality of foreign investment was the British economist Dunning. 
Dunning argues that the investment motives of multinational firms and the location advantages of the host country determine the quality of foreign investment [9]. Jin et al. further argue that the competitiveness stage of the host country plays a decisive role in the quality of international investment [10]. In addition, some scholars have explored the factors influencing the quality of foreign investment in terms of infrastructure level, technology level and competitiveness, and preferential policies, respectively [11]. In recent years, as the scale of foreign investment attracted by China has been expanding (see Figure 2), scholars have begun to pay attention to the quality of foreign investment utilized in China and come to different conclusions. Fu conducted an empirical study based on the average size of the project, the proportion of large and medium-sized enterprises, and other indicators and concluded that the quality of China's use of foreign investment is at a low level [12]. Nei and Liu, on the contrary, combined panel data from 2003-2015 in China for an empirical analysis and concluded that the productivity improvement of local firms significantly improved the quality of foreign investment in China [13].

In summary, the relevant literature on the impact of new city construction on the quality of foreign investment is still scarce, and scholars have not yet focused on the impact of smart cities on the quality of attracting foreign investment in China. As an important national "policy experiment," the smart city construction pilot is a remedy for the bottleneck of urbanization development. The pilot policy will lead to changes in the institutional environment, releasing dividends through the policy and thus attracting large-scale FDI inflows. As a reflection of China's institutional innovation, the smart city pilot can effectively improve the efficiency of urban management and enhance the supply of urban industries and services, promote the upgrading of urban industrial structure, and thus create a first-class business environment and expand the attractiveness to foreign enterprises. Therefore, based on the above literature review, this article proposes the core theoretical hypothesis to be tested: the construction of smart city pilot cities can improve the quality of foreign investment.

\section{Materials and Methods}

3.1. Measurement Methods. Taking into account the variability of economic agents before and after the smart city pilot policy, the variability of the provinces to which different cities belong and their own scale of attracting FDI, and the variability of being influenced by different levels of economic development, this article uses the smart city pilot in China as a quasi-natural experiment to analyze the impact of the smart city pilot on the quality of FDI using a difference-in-difference (DID) model. The DID model can effectively correct for "policy endogenous" and "selectivity bias" in the policy evaluation process. Among them, the double difference mainly comes from two levels, city and year, comparing the quality of foreign investment in pilot cities and nonpilot cities before and after the pilot. Under the model setting, this article sets the following DID model based on the study of Gehrsitz [14].

$\mathrm{FDI}_{c t}$ denotes FDI quality, and the subscripts $c$ and $t$ denote city as well as time, respectively. Smart ${ }_{c t}$ indicates whether city $c$ is a pilot city in period $t$ after the start of the smart city pilot. If Smart $c t=1$, then it means that city $c$ is a smart city pilot city in period $t$. Conversely, if $\operatorname{Smart}_{c t}=0$ means that city $c$ is not a smart city pilot city in period $t . D_{c t}$ denotes a set of control variables. $W_{t}$ and $W_{c}$ denote the time fixed effect as well as the city fixed effect, respectively. $\vartheta_{c t}$ denotes the error term:

$$
\mathrm{FDI}_{c t}=\alpha_{0}+\alpha_{1} \operatorname{Smart}_{c t}+\alpha_{2} D_{c t}+W_{t}+W_{c}+\vartheta_{c t} .
$$

\subsection{Samples and Variables. China's Ministry of Housing and} Urban-Rural Development and Ministry of Science and Technology announced three batches of smart city pilot lists in 2012, 2013, and 2014, respectively. For the purpose of model construction, after considering the consistency and availability of data, the prefecture-level cities with serious data deficiencies were excluded from this article, and data from 226 prefecture-level cities from 2006-2017 were selected to assess the impact of smart city pilots on the quality of FDI. Among them, the pilot cities of smart cities belong to the processing group, and the pilot cities not included in the "smart cities" belong to the control group. The data in this article are obtained from official statistical sources such as China City Yearbook and China Statistical Yearbook. Table 1 indicates that the variables selected in this article do not have serious autocorrelation.

3.2.1. Explained Variable: Quality of FDI. Currently, there is no unified standard in the academic community for measuring the quality of foreign investment. In this article, the average size of FDI enterprises and the foreign investment performance index are used to refer to the quality of FDI after referring to the studies of scholars such as Zhong and Chen and Sun and Zhou, respectively $[15,16]$. (1) Average size of foreign-invested enterprises $\left(\mathrm{fdi}_{1}\right)$ : they are calculated using the ratio of actual FDI to the number of foreigninvested enterprises in each prefecture-level city. (2) Foreign investment performance index: this was calculated using the following equation:

$$
\mathrm{fdi}_{2}=\frac{\left(\mathrm{fdi}_{c t} / \mathrm{cnfdi}_{t}\right)}{\left(\operatorname{gdp}_{c t} / \mathrm{cngdp}_{t}\right)},
$$

where $\mathrm{fdi}_{c t}$ denotes the amount of actual FDI acquired by city $c$ in year $t$, cnfdi $_{t}$ denotes the actual FDI acquired by China in year $t, \operatorname{gdp}_{c t}$ denotes the gross regional product of

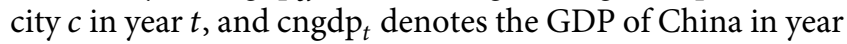
$t$.

3.2.2. Explanatory Variable: Whether It Is a "Smart Pilot" City. The core variable interpretation of a smart city pilot (smart) is defined as taking a value of 1 for the year of pilot and subsequent years if the city implements a smart city pilot effort, and 0 otherwise. Since the smart city pilots were 

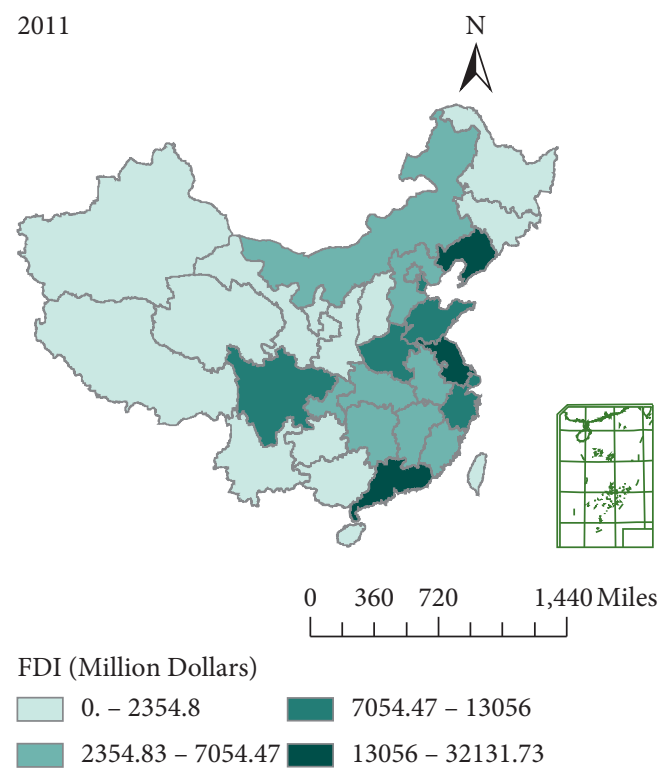

(a)

2017

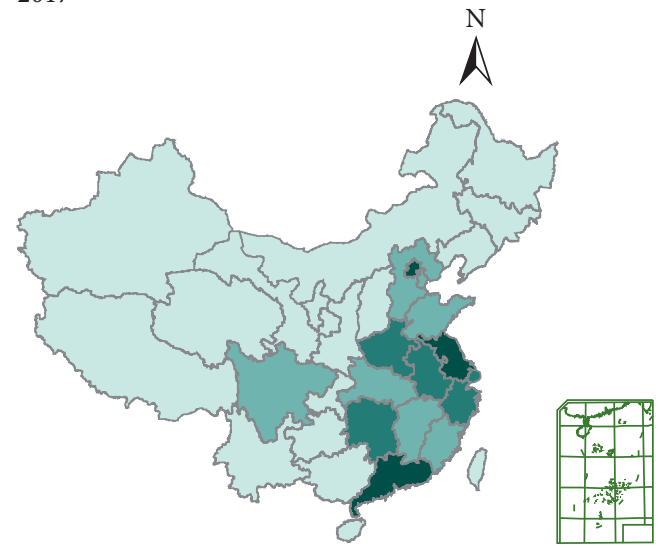

$0 \quad 350 \quad 700$

FDI (Million Dollars)
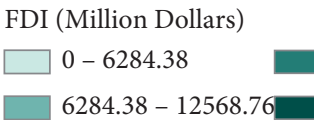
$12568.76-18853.14$

$18853.14-25137.5$

(c)
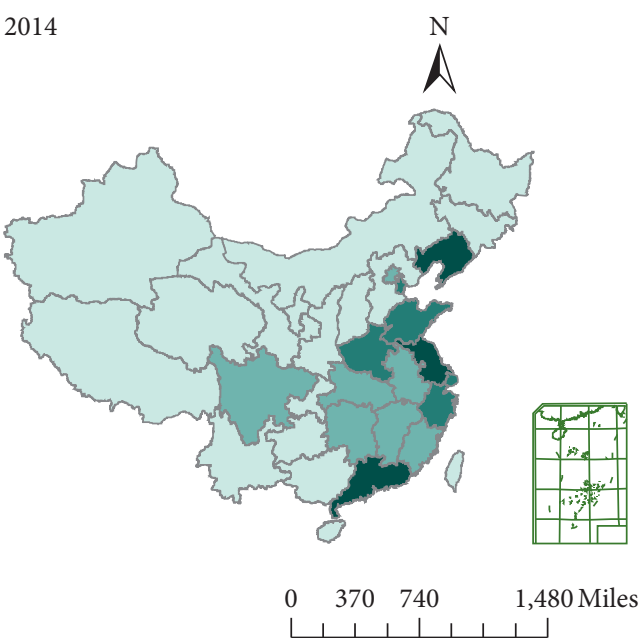

FDI (Million Dollars)

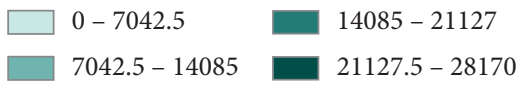

(b)

2019

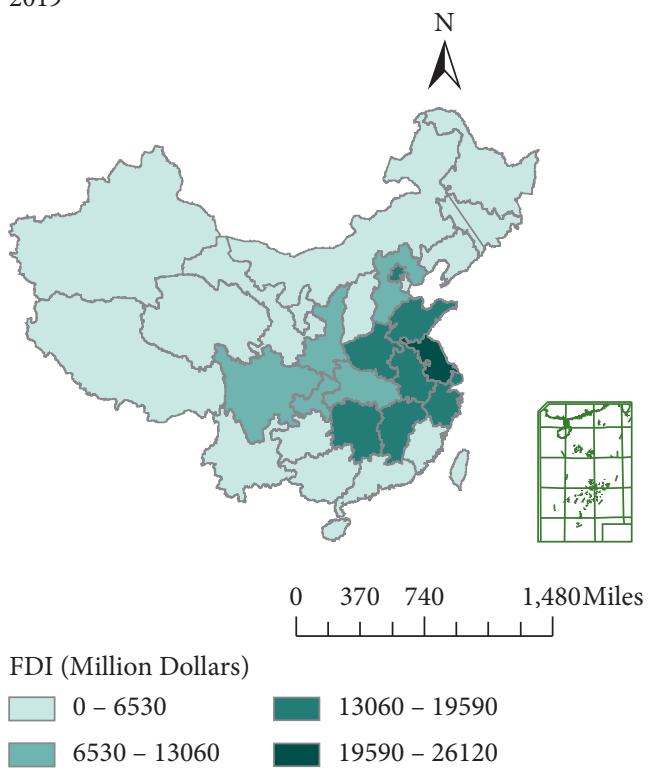

(d)

FIGURE 2: Changes in trends in attracting foreign investment by province (missing data for individual provinces). (a) 2011 . (b) 2014 . (c) 2017. (d) 2019 .

TABle 1: Correlation coefficient matrix.

\begin{tabular}{|c|c|c|c|c|c|c|c|c|c|c|c|}
\hline & LNGDP & RPOP & LNJOB & LNROAD & LNEDU & SAVERATE & LNSO2 & $\mathrm{CTCH}$ & LNGFDI & LNTAX & LIFE \\
\hline LNGDP & 1 & & & & & & & & & & \\
\hline RPOP & 0.247 & 1 & & & & & & & & & \\
\hline LNJOB & 0.459 & 0.136 & 1 & & & & & & & & \\
\hline LNROAD & 0.834 & 0.473 & 0.418 & 1 & & & & & & & \\
\hline LNEDU & 0.731 & -0.04 & 0.418 & 0.610 & 1 & & & & & & \\
\hline SAVERATE & 0.266 & 0.412 & 0.344 & 0.385 & 0.295 & 1 & & & & & \\
\hline LNSO2 & 0.293 & -0.05 & 0.148 & 0.239 & 0.294 & -0.02 & 1 & & & & \\
\hline $\mathrm{CTCH}$ & 0.388 & 0.385 & 0.197 & 0.379 & 0.312 & 0.383 & 0.037 & 1 & & & \\
\hline LNGFDI & 0.803 & 0.327 & 0.359 & 0.760 & 0.524 & 0.270 & 0.257 & 0.328 & 1 & & \\
\hline LNTA & 0.848 & 0.135 & 0.308 & 0.666 & 0.561 & 0.064 & 0.305 & 0.254 & 0.723 & 1 & \\
\hline LIFE & 0.328 & 0.161 & 0.104 & 0.288 & 0.058 & 0.052 & 0.017 & 0.106 & 0.265 & 0.302 & 1 \\
\hline
\end{tabular}


conducted a total of three times from 2012-2014, the sample in this paper covers all batches of the smart city pilots.

3.2.3. Control Variables. In this article, based on the studies of Moraghen et al., Nie, Ang-Tan and Ang, and Doytch and Eren, the following control variables were selected $[8,17-19]$. The logarithm of the gross regional product is used to measure the level of economic development (LNGDP). The level of urbanization (RPOP) is measured using the ratio of the total year-end population of a city's municipal area (CPOP) to the total year-end population of the city (POP). The logarithm of the average number of workers on the job is used to measure the size of the labor force (LNJOB). The logarithm of the actual paved road area at the end of the year is used to measure the level of infrastructure development (LNROAD). The logarithm of the number of educational practitioners is used to measure the level of human capital (LNEDU). The savings' rate (SAVERATE) is measured using the ratio of the balance of various RMB deposits in financial institutions (SAVE) to GDP at the end of the year. The logarithm of industrial SO2 emissions is used to measure the urban environment (LNSO2). The level of science, technology, and innovation $(\mathrm{CTCH})$ is measured using the ratio of the total number of personnel in the research integrated technical services' sector (TCH) to the total urban population. The logarithm of the total industrial output value of foreign-invested industrial enterprises above the scale (Annual revenue from main business greater than $\$ 3.08$ million) is used to measure the degree of openness to the outside world (LNGFDI). Business environment is measured by the logarithm of total profit tax (LNTAX). Environmental governance capacity is measured by the ratio of household waste disposal without harm (LIFE).

\section{Results and Discussion}

4.1. Parallel Trend Test. When using DID, an important prerequisite is to satisfy that the treatment and control groups are trending in parallel [20]. Since China's smart city pilot is conducted in batches, this article uses $\mathrm{fdi}_{2 c t}$ as the explanatory variable and selects the first batch of smart city pilot list in 2012 for parallel trend testing, excluding the pilot cities in 2013 and 2014. In this article, we refer to Beck et al. and set up the following model for parallel trend testing [21]:

$$
\begin{aligned}
\mathrm{fdi}_{2 c t}= & \alpha+\beta_{1} \text { smart }_{t-5}+\beta_{2} \text { smart }_{t-4}+\beta_{3} \text { smart }_{t-3}+\cdots+\beta_{8} \text { smart }_{t+2} \\
& +\beta_{9} \text { smart }_{t+3}+\gamma D_{c t}+W_{t}+W_{c}+\vartheta_{c t} .
\end{aligned}
$$

In equation (3), smart $_{t+n}$ denotes the dummy variables for $n$ years before and after the launch of the smart city pilot, respectively. Using the year 2012 as the boundary for the first batch of pilot work, we examined the trend of fdi ${ }_{2}$ in the first five years and the last three years of the pilot. If the coefficient of smart $_{t-n}$ is not significant and the coefficient of smart $_{t+n}$ is significant, it indicates that the treatment group has a parallel trend with the control group and the driving effect of the smart city pilot work is obvious. The results of the parallel trend test are shown in Figure 3. The coefficient of smart $t_{-n}$ was not significant before the smart city pilot was launched, and the coefficient was only significantly positive after the smart city pilot was launched, which indicates that the model passed the parallel trend test and the policy effect of the smart city pilot has a certain degree of persistence.

4.2. Baseline Regression Results. Table 2 shows the regression results of smart city pilot on the quality of FDI. Among them, (1) and (2) are the regression results with the scale of foreign-invested enterprises $\left(\mathrm{fdi}_{1}\right)$ and the performance index of foreign investment $\left(\mathrm{fdi}_{2}\right)$ as the explanatory variables, respectively, (3) and (4) are the regression results with the control variables added. We find that the estimated coefficients for the smart city pilots are significantly positive after controlling city fixed effects as well as time fixed effects, regardless of the inclusion or exclusion of control variables. Exploring columns (3) and (4) reveals that the estimated coefficients of smart $c$ are 0.249 and 0.178 , respectively, which indicate that compared to nonpilot cities, the average size of foreign-invested enterprises and the foreign investment performance index of smart cities are on average 0.249 units and 0.178 units higher, respectively. Therefore, this indicates that the smart city pilot has indeed helped to improve the quality of FDI and played the expected promotional role.

For the estimation results of the control variables, we analyze them in columns (3) and (4). In column (3), the coefficient of LNGFDI is significantly positive at the $5 \%$ level, which indicates that foreign-invested enterprises prefer to invest in cities with higher levels of openness to the outside world and thus expand their enterprises. In column (4), the coefficient of LNGDP is significantly negative at the $10 \%$ level, indicating that the level of urban economic development significantly and negatively affects the level of performance of foreign-invested enterprises. The possible reason is that, in the more economically developed cities, there are more local enterprises of the same type as foreign-invested enterprises, which intensifies the competitive behavior among enterprises and does not help foreign enterprises to improve their performance level. The LNROAD is significantly positive in both column (3) and column (4), indicating that the level of infrastructure development significantly and positively affects the quality of foreign investment, which is consistent with the findings of $\mathrm{He}$ et al. [22].

4.3. Robustness Tests. Based on the studies of Cheng et al. and Liang, this article uses $\mathrm{fdi}_{2}$ as the explanatory variable for robustness testing $[23,24]$. Three methods are used: 


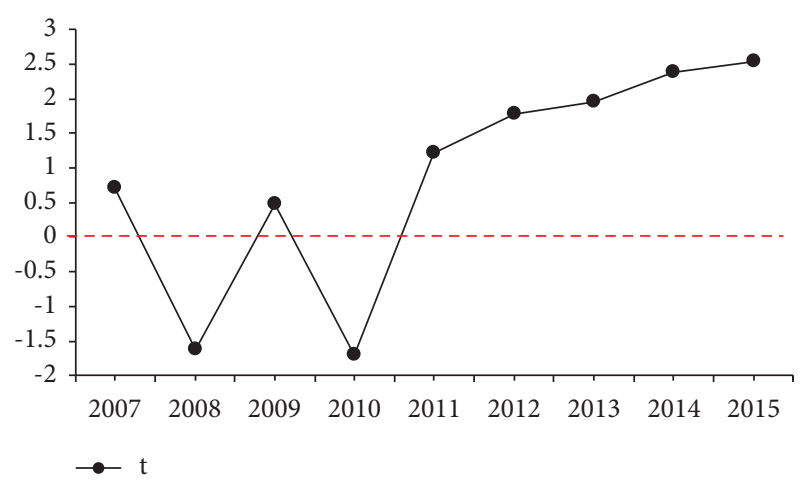

Figure 3: Parallel trend check chart.

TABLE 2: Estimated results of the baseline regression.

\begin{tabular}{|c|c|c|c|c|}
\hline Variables & $(1)$ & $(2)$ & (3) & (4) \\
\hline SMART & $\begin{array}{c}0.168^{* *} \\
(2.10)\end{array}$ & $\begin{array}{c}0.134^{* *} \\
(2.25)\end{array}$ & $\begin{array}{c}0.249^{* * * *} \\
(2.73)\end{array}$ & $\begin{array}{c}0.178^{* *} \\
(2.57)\end{array}$ \\
\hline LNGDP & & & $\begin{array}{l}-0.288 \\
(-0.94) \\
\end{array}$ & $\begin{array}{c}-0.492^{* *} \\
(-2.11) \\
\end{array}$ \\
\hline RPOP & & & $\begin{array}{c}-0.0494 \\
(-0.16)\end{array}$ & $\begin{array}{c}-0.0372 \\
(-0.15)\end{array}$ \\
\hline LNJOB & & & $\begin{array}{l}-0.104 \\
(-0.68)\end{array}$ & $\begin{array}{l}-0.104 \\
(-0.90)\end{array}$ \\
\hline LNROAD & & & $\begin{array}{c}0.260^{* *} \\
(2.13)\end{array}$ & $\begin{array}{c}0.236^{* *} \\
(2.54)\end{array}$ \\
\hline LNEDU & & & $\begin{array}{l}-0.250 \\
(-0.78)\end{array}$ & $\begin{array}{c}0.0390 \\
(0.16)\end{array}$ \\
\hline SAVERATE & & & $\begin{array}{c}0.0874 \\
(0.83)\end{array}$ & $\begin{array}{c}0.0373 \\
(0.47) \\
\end{array}$ \\
\hline LNSO2 & & & $\begin{array}{c}0.00916 \\
(0.18)\end{array}$ & $\begin{array}{c}-0.0500 \\
(-1.29)\end{array}$ \\
\hline $\mathrm{CTCH}$ & & & $\begin{array}{l}-0.778 \\
(-0.10)\end{array}$ & $\begin{array}{l}-0.782 \\
(-0.14)\end{array}$ \\
\hline LNGFDI & & & $\begin{array}{c}0.171^{* * *} \\
(2.82)\end{array}$ & $\begin{array}{c}0.0102 \\
(0.22)\end{array}$ \\
\hline LNTAX & & & $\begin{array}{c}0.0716 \\
(1.43) \\
\end{array}$ & $\begin{array}{c}0.0224 \\
(0.59) \\
\end{array}$ \\
\hline LIFE & & & $\begin{array}{c}-0.000502 \\
(-0.40)\end{array}$ & $\begin{array}{c}-0.000185 \\
(-0.20)\end{array}$ \\
\hline Cons_ & $\begin{array}{c}0.0631 \\
-0.88 \\
\end{array}$ & $\begin{array}{c}0.153^{* * *} \\
(2.86) \\
\end{array}$ & $\begin{array}{l}6.971 \\
(1.38) \\
\end{array}$ & $\begin{array}{c}6.320^{*} \\
(1.65)\end{array}$ \\
\hline City fe & YES & YES & YES & YES \\
\hline Year fe & YES & YES & YES & YES \\
\hline$N$ & 2712 & 2712 & 2712 & 2712 \\
\hline$R^{2}$ & 0.022 & 0.048 & 0.029 & 0.050 \\
\hline
\end{tabular}

Notes: $T$ statistics are in parentheses; ${ }^{*} p<0.10 ;{ }^{* *} p<0.05 ;{ }^{* * *} p<0.01$.

front-loading the smart city pilot to 2011, excluding extreme values and lagging the smart city pilot variable, and all control variables by one period.

4.3.1. Front-Loading Smart City Pilot to 2011. To ensure randomness in the enactment of the three batches of smart city pilot policies from 2012-2014, a counterfactual test was conducted by changing the timing of the implementation of the smart city pilots. For the quality of FDI, apart from the smart city pilot work, other policies such as the "low carbon city" pilot policy and consequent factors may cause differences in the quality of FDI that are not generated by the implementation of the smart city pilot work, which would lead to the previous conclusion not being valid. Therefore, in this article, all three batches of smart city pilots are front loaded to 2011 for regression, and if the coefficient of smart ${ }_{c t}$ estimation is still significantly positive, it indicates that the variation in the quality of FDI may originate from the influence of other policies or random factors. If the coefficient of smart ${ }_{c t}$ is insignificant or significantly negative, it proves that the enactment of the smart city pilot is random. The results are shown in (1) in Table 3, where the coefficient of smart $_{c t}$ is insignificant, which indicates that the estimation results of this article possess good robustness.

4.3.2. Excluding Extreme Values. As shown in Table 4, the standard deviation data between the explanatory variables indicate that the samples differ significantly between the variables and that there are extreme end outliers. Therefore, this article adopts the "tailing method" to deal with extreme outliers, in which the highest and lowest $1 \%$ of all the control variables are reduced, and the reduced samples are regressed. The results are shown in (2) in Table 3, where the coefficient on smart ${ }_{c t}$ remains significantly positive, proving that smart city pilots significantly and positively contribute to the quality of FDI.

\subsubsection{Lagging the Smart City Pilot Variable and All Control} Variables by One Period. Considering the time lag of the smart city pilot, this article treats smart ${ }_{c t}$ with a one-period lag, while all control variables are also treated with a oneperiod lag to avoid joint cubic equation bias. The treated regression results are shown in (3) in Table 3 . The coefficient of smart $c t$ is still significantly positive, proving that the findings of this article are robust.

4.4. Placebo Test. For the DID model constructed in this article, there may be differences in the characteristics of the treatment group and the control group other than whether they are smart city pilot cities before the smart city pilot work is carried out. For equation (1), although it has controlled most of the city characteristics variables, it is not sufficient to control all city characteristics. Therefore, in this article, we refer to Yu et al. and adopt the following method of placebo testing with $\mathrm{fdi}_{2}$ as the explanatory variable to observe whether omitted characteristics may have an effect on the results [25]. First, the expression for the $\mathrm{fdi}_{2}$ coefficient is derived based on equation (1):

$$
\alpha_{1 r}=\alpha_{1}+\delta * \frac{\operatorname{cov}\left(\operatorname{smart}_{c t}, \vartheta_{c t} \mid D\right)}{\operatorname{var}\left(\operatorname{smart}_{c t}, D\right)} .
$$

If a variable can be found to replace smart ${ }_{c t}$, this variable does not theoretically affect the corresponding (i.e. $\alpha_{1}=0$ ); after estimating $\alpha_{1 r}=0$, it can be proved that $\delta=0$; i.e., the omitted feature does not affect the estimation result. 
TABLE 3: Robustness tests.

\begin{tabular}{lccc}
\hline Variables & $(1)$ & $(2)$ & $(3)$ \\
\hline \multirow{2}{*}{ Smart } & 0.182 & $0.156^{*}$ & $0.188^{*}$ \\
& $(1.86)$ & $(2.24)$ & $(2.45)$ \\
\hline Control variables & YES & YES & YES \\
City fe & YES & YES & YES \\
Year fe & YES & YES & YES \\
$R^{2}$ & 0.0589 & 0.0521 & 0.0529 \\
\hline
\end{tabular}

Notes: $T$ statistics are in parentheses; ${ }^{*} p<0.10 ;{ }^{* *} p<0.05 ;{ }^{* * *} p<0.01$.

TABLE 4: Summary statistics.

\begin{tabular}{lccccc}
\hline Variables & Units & Mean & Sd & Min & Max \\
\hline FDI1 & $100,000,000$ & 0.239 & 1.151 & 0 & 33.33 \\
FDI2 & 1 & 0.272 & 0.864 & 0 & 19.82 \\
SMART & 1 & 0.209 & 0.406 & 0 & 1 \\
LNGDP & 10,000 & 16.27 & 0.926 & 13.91 & 19.55 \\
RPOP & 1 & 0.34 & 0.23 & 0.43 & 1 \\
LNJOB & 10,000 & 3.164 & 1.521 & -2.74 & 6.625 \\
LNROAD & 10,000 & 6.986 & 0.92 & 4.127 & 9.975 \\
LNEDU & 1 & 10.71 & 0.652 & 8.455 & 13.13 \\
SAVERATE & 1 & 1.024 & 0.76 & 0.214 & 9.054 \\
LNSO2 & 1,000 & 10.56 & 1.137 & 0.693 & 14.15 \\
CTCH & 1 & 0.002 & 0.004 & 0 & 0.145 \\
LNGFDI & 10,000 & 13.64 & 1.859 & 7.324 & 18.82 \\
LNTAX & 10,000 & 13.77 & 1.35 & 6.56 & 17.77 \\
LIFE & 1 & 0.86 & 0.23 & 0 & 3.62 \\
\hline
\end{tabular}

Therefore, this article regresses the shock of the smart city pilot after becoming random and repeats this random regression process 1000 times so that $\mathrm{fdi}_{2 c t}$ will not be affected (i.e., $\alpha_{1 r}^{\text {random }}=0$ ). The results are shown in Figure 4 , and we find that the distributions of $\alpha_{1 r}^{\text {random }}$ are all around $x=0$ and normally distributed after 1000 estimations, which indicates that $\delta=0$ holds, i.e., the estimation results in this article are reasonable.

4.5. Heterogeneity Analysis. Different cities have certain differences in economic structure, policy implementation, and natural endowment degrees, and these differences may lead to different effects of smart city pilot policies on different cities, which in turn affects the quality of FDI. Therefore, this article draws on Doytch et al and Li and Li to further test regional differences in the quality of foreign investment affected by smart city pilot policies, using $\mathrm{fdi}_{2}$ as the explained variable $[26,27]$.(1) Divide cities into three equal parts based on 2016 GDP per capita. Cities with low economic development have GDP per capital in the $0 \%-33 \%$ range. Medium economic development level cities have GDP per capital in the 33\%-66\% range. Cities with high economic development have GDP per capital in the $66 \%$ or higher range. (2) Heterogeneity analysis was performed by two methods based on the geographic location of the city and divided into three subsamples: eastern, central, and western. (3) The degree of urban natural resource dependence was determined based on the proportion of mining

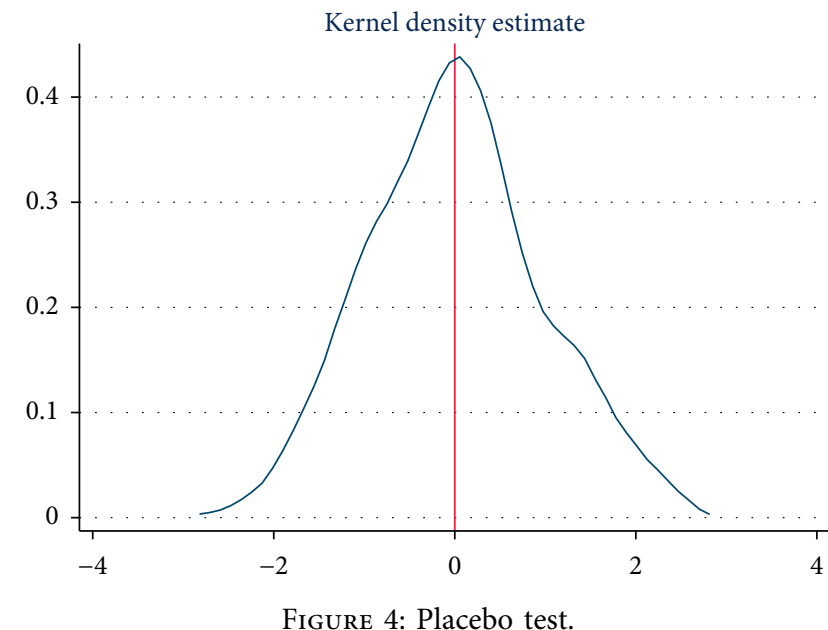

employment to total urban employment in 2016 and was categorized into three levels: cities with low natural resource dependency are in the $0 \%-33 \%$ range, cities with medium natural resource dependency are in the 33\%-66\% range, and cities with high natural resource dependency are in the $66 \%$ range.

As can be seen from Table 5, the improvement in the quality of FDI in the smart city pilot is more significant in the subsample of western cities. This may be due to the fact that the economic conditions and FDI levels of western cities are relatively backward compared to those of eastern and central cities. When the smart city pilot policy is implemented, the level of infrastructure development within the region can be improved, which can attract a large amount of foreign capital and high-quality foreign enterprises, thus improving the quality of FDI in western cities. Meanwhile, the enhancement of quality of FDI by smart city pilot is more significant in the subsample of cities with low natural endowment dependence. This is because the reduction of natural resource dependence will increase the level of physical investment, education, and $\mathrm{R} \& \mathrm{D}$ in the region, which is more in line with the trend of foreign-invested enterprises moving from resourceseeking to market-seeking, efficiency-seeking, and system-seeking, which enables foreign-invested enterprises to utilize the agglomeration effect and better play the promotion effect of smart city construction on the quality improvement of FDI. 
TABLE 5: Heterogeneity.

\begin{tabular}{lccccccccc}
\hline \multirow{2}{*}{ Variables } & \multicolumn{3}{c}{ Geographical position } & \multicolumn{3}{c}{ Economic development } & \multicolumn{3}{c}{ Natural resource dependency } \\
& $(1)$ & $(2)$ & $(3)$ & $(4)$ & $(5)$ & $(6)$ & $(7)$ & $(8)$ & $(9)$ \\
& East & Middle & West & Low & Medium & High & Low & Medium & High \\
\hline \multirow{2}{*}{ Smart } & 0.0968 & 0.216 & $0.0742^{* *}$ & -0.0947 & 0.172 & 0.190 & $0.386^{* *}$ & 0.0278 & 0.103 \\
& $(0.103)$ & $(1.50)$ & $(2.66)$ & $(-0.54)$ & $(1.77)$ & $(1.95)$ & $(3.00)$ & $(0.31)$ & $(0.76)$ \\
\hline Control variables & YES & YES & YES & YES & YES & YES & YES & YES & YES \\
City Fe & YES & YES & YES & YES & YES & YES & YES & YES & YES \\
Year Fe & YES & YES & YES & YES & YES & YES & YES & YES & YES \\
R2 & 0.0986 & 0.0634 & 0.1105 & 0.932 & 0.1035 & 0.0482 & 0.0976 & 0.0704 & 0.0540 \\
\hline
\end{tabular}

Notes: $T$ statistics are in parentheses; ${ }^{*} p<0.10 ;{ }^{* *} p<0.05 ;{ }^{* * *} p<0.01$

\section{Conclusions}

Based on data from 226 prefecture-level cities from 2006-2017, this article explores whether smart city pilots, which were gradually introduced in 2012 as a quasi-natural experiment, have improved the quality of foreign investment in China using a DID model after controlling fixed effects at both the time and city levels. The results show that the core explanatory variable smart city pilot has a significant positive effect on the quality of foreign investment as indicated by the average size of foreign-invested enterprises and the foreign investment performance index at two levels, while the level of infrastructure development among the control variables has a significant positive effect on the quality of foreign investment and the level of wages has a significant negative effect on the quality of foreign investment. After parallel trend tests, a series of robustness tests, and placebo tests, the results are still held. In addition, heterogeneity analysis shows that smart city pilot has a significant contribution to improve the quality of FDI in western cities and cities with low natural resource dependence.

Although the pilot work on smart cities has significantly improved the quality of attracting foreign investment in China, China and other countries around the world should continue to rationalise and improve the construction of smart cities in the following 3 areas to meet the optimisation and sustainable development of foreign investment in the postepidemic era.

First, China should further liberalise the market access conditions for infrastructure in smart city pilot cities in western cities of China, treating foreign investment equally. The empirical results show that the smart city construction has improved the quality of foreign investment in western cities, more significantly compared to eastern and central cities. Therefore, for the western cities whose economic development level is still at a low level, they should further liberalise the market access in the field of infrastructure investment, expand the channels for foreign enterprises to participate in construction investment with the help of smart city construction, give full play to the decisive role of the market for resource allocation, promote the healthy and orderly operation of the market, and promote the highquality development of the region's urban economy.

Second, governments should regulate and promote PPP financing models to bring in foreign investment and pay attention to scientific planning and step-by-step implementation. Currently, smart city construction focuses on seven important areas of new infrastructure: $5 \mathrm{G}$ base stations, data centers, industrial Internet, artificial intelligence, charging piles, extrahigh voltage, and high-speed rail and urban rail transit. In terms of the investment scale, the market size of the seven new infrastructure areas still has room to rise and will still need strong capital as support in the future. The government can only introduce high-quality foreign investment to improve efficiency by standardizing and continuously developing the PPP financing model.

Third, countries around the world should strengthen data management and security to break information silos and reduce network risks. In the construction of smart city, the phenomenon of fragmentation is very common, the massive amount of data failed to achieve effective integration, and network security risks are increasing. Optimizing the foreign investment environment requires not only hard new infrastructure but also soft new infrastructure, the most urgent of which is the construction of urban data. Only to carry out a comprehensive evaluation of urban big data, realize the standardized management of the whole procedure from data collection to data capitalization, and implement basic systems such as grade protection and security measurement, can we effectively guarantee the security of foreign investment in the process of sharing and using urban data and reduce network risks?

\section{Data Availability}

The processed data used to reproduce the findings of the study cannot be shared at this time as the data also form part of an ongoing study.

\section{Conflicts of Interest}

The authors declare no conflicts of interest.

\section{Authors' Contributions}

C.Y., Z.Z., and J.C. conceptualized the study; C.Y. and Z.Z. developed the methodology; Z.Z. helped with software; C.Y., Z.Z., and J.C. validated the study; C.Y., Z.Z., and J.C. carried out formal analysis; C.Y. investigated the study; Z.Z. helped with the resources; Z.Z. curated the data; Z.Z. and J.C. wrote and prepared the original draft; C.Y., Z.Z., and J.C. reviewed and edited the article; C.Y. visualized the study; C.Y. 
supervised the study; C.Y. administrated the project; C.Y. carried out funding acquisition. All authors have read and agreed to the published version of the manuscript.

\section{Acknowledgments}

This research was funded by National Social Science Foundation Projects (Grant no. "19FJYB043").

\section{References}

[1] L. Alcaide Muñoz and M. P. Rodríguez Bolívar, "Different levels of smart and sustainable cities construction using e-participation tools in European and central asian countries," Sustainability, vol. 13, no. 6, p. 3561, 2021.

[2] A. Parlina, K. Ramli, and H. Murfi, "Exposing emerging trends in smart sustainable city research using deep autoencoders-based fuzzy C-means," Sustainability, vol. 13, no. 5 , p. 2876, 2021.

[3] L. Linde, D. Sjödin, V. Parida, and J. Wincent, "Dynamic capabilities for ecosystem orchestration A capability-based framework for smart city innovation initiatives," Technological Forecasting and Social Change, vol. 166, no. 1, Article ID 120614, 2021.

[4] L. Shi, C. Shi, X. Wu, and L. Ma, "Accelerating the development of smart city initiatives amidst the COVID-19 pandemic: the case of health code in China," Journal of Asian Public Policy, no. 8, pp. 1-18, 2021.

[5] X. Zhou and L. Li, "Can smart city construction become a new driving force for economic growth?" Economic Warp and Woof, vol. 37, no. 6, pp. 10-17, 2020.

[6] Y. Tang, "Digital economy enables high quality development of cities--quasi natural experimental analysis based on smart city construction," Price Theory and Practice, vol. 9, pp. 156-180, 2020.

[7] Y. Shi and X. Zhang, "Does smart city construction promote financial development--quasi natural experimental research based on the establishment of smart city," Finance and Economy, vol. 12, pp. 82-88, 2019.

[8] F. Nie, "The impact of National Smart City pilot on the "dual margin" expansion of FDI: theoretical mechanism and empirical analysis," International Trade Issues, vol. 10, pp. 84-99, 2019.

[9] J. H. Dunning, "The eclectic paradigm of international production," 1988.

[10] C. Jin, S. O. Business, and N. University, "Relationships among foreign direct investment, economic development level and environmental pollution: an empirical study based on panel data of provinces of China from 2003 to 2013," Journal of Tianjin Normal University(Natural Science Edition), no. 5, 2017.

[11] D. Qian and B. I. E. Zhaoxia, "FDI, human resource accumulation and economic growth, economic research," no. 4, , 2006.

[12] Y. Fu, "An empirical study on the quality of FDI in China," Statistical research, vol. 10, pp. 9-17, 2008.

[13] F. Nie and H. Liu, "The impact of national innovative city construction on the quality of FDI in China," Economic Review, vol. 6, pp. 67-79, 2019.

[14] M. Gehrsitz, "The effect of low emission zones on air pollution and infant health," Journal of Environmental Economics and Management, vol. 83, pp. 121-144, 2017.
[15] X. Zhong and X. Chen, "Comparative study on regional differences and influencing factors of foreign direct investment," Statistical Research, vol. 29, no. 3, pp. 54-60, 2012.

[16] L. Sun and k. Zhou, "Research on the impact of China's low carbon pilot policy on the quality of Foreign Direct Investment--quasi natural experimental evidence from the construction of "low carbon city," Southeast Academic Journal, vol. 4, pp. 136-146, 2020.

[17] W. Moraghen, B. Seetanah, and N. Sookia, "The impact of exchange rate and exchange rate volatility on mauritius foreign direct investment: a sectorise analysis," International Journal of Finance \& Economics, vol. 1, no. 1, 2020.

[18] R. Ang-Tan and S. Ang, "Understanding the smart city race between Hong Kong and Singapore," Public Money \& Management, vol. 99, no. 1, pp. 1-10, 2021.

[19] N. Doytch and M. Eren, "Institutional determinants of sectoral FDI in eastern European and central asian countries: the role of investment climate and democracy," Emerging Markets Finance and Trade, vol. 48, pp. 14-32, 2012.

[20] S. Chen, Y. Cao, and J. Li, "The effect of water rights trading policy on water resource utilization efficiency: evidence from a quasi-natural experiment in China," Sustainability, vol. 13, no. 9 , p. 5281, 2021.

[21] T. Beck, R. Levine, and A. Levkov, "Big bad banks? the winners and losers from bank deregulation in the United States," The Journal of Finance, vol. 65, no. 5, pp. 1637-1667, 2010.

[22] W. Song, "Research on wage level and location change of Foreign Direct Investment," Research on Financial Issues, vol. 10, pp. 48-54, 2013.

[23] J. Cheng, J. Yi, S. Dai, and Y. Xiong, "Can low-carbon city construction facilitate green growth? Evidence from China's pilot low-carbon city initiative," Journal of Cleaner Production, vol. 231, no. 9, pp. 1158-1170, 2019.

[24] F. H. Liang, "Does foreign direct investment harm the host country's environment? Evidence from China," Current Topics in Management, vol. 17, pp. 105-121, 2014.

[25] C. Yu, R. Zhang, L. An et al., "Has China's belt and road initiative intensified bilateral trade links between China and the involved countries?" Sustainability, vol. 12, 2020.

[26] N. Doytch, R. U. Mendoza, and C. I. Siriban, "Does mining FDI crowd in other investments? investigation of FDI intersectoral linkages," Comparative Economic Studies, vol. 57, no. 2, pp. 326-344, 2015.

[27] W. H. Li and N. Li, "Natural resource endowment, marketbased allocation and industrial structural transformationempirical evidence from 116 resource-based cities," Modern Economic Research, vol. 8, pp. 52-63, 2021. 